Meta

Journal des tradlucteurs

Translators' Journal

\title{
L'A.B.C. de l'adaptation publicitaire
}

\section{Roger Boivineau}

Volume 17, numéro 1, mars 1972

L’adaptation publicitaire

URI : https://id.erudit.org/iderudit/002097ar

DOI : https://doi.org/10.7202/002097ar

Aller au sommaire du numéro

Éditeur(s)

Les Presses de l'Université de Montréal

ISSN

0026-0452 (imprimé)

1492-1421 (numérique)

Découvrir la revue

Citer cet article

Boivineau, R. (1972). L'A.B.C. de l'adaptation publicitaire. Meta, 17(1), 5-28.

https://doi.org/10.7202/002097ar d'utilisation que vous pouvez consulter en ligne.

https://apropos.erudit.org/fr/usagers/politique-dutilisation/ 


\section{$L^{\prime}$ a.b.c. de l'adaptation publicitaire}

* La publicité, mais cela ne devrait pas se traduire ! *

La phrase mise ici en exergue pourrait être une boutade. En fait, c'est une réflexion teintée d'indignation, spontanément exprimée par l'un des étudiants en traduction à qui on exposait le programme du cours d'adaptation publicitaire donné à l'Université de Montréal.

Déjà au fait de certains problèmes de la traduction, mais n'ayant aucune connaissance particulière de la publicité, si ce n'est celle que peut en posséder l'homme de la rue, ce garçon avait décelé ce qu'il peut souvent y avoir de laborieux, d'illogique, et même d'irréalisable, dans l'entreprise qui consiste à faire passer le contenu d'un texte publicitaire d'une langue dans une autre.

Pourtant, on le verra ailleurs dans ce numéro de META, l'adaptation domine la production publicitaire de langue française au Canada; elle est monnaie courante dans d'autres pays bilingues ou multilingues, et n'est pas absente de la publicité de nombreux pays unilingues. Il y a là une situation de fait dont les causes sont, on le verra également, presque uniquement économiques. Situation regrettable, mais qui, espérons-le, évoluera favorablement au Québec avec le développement, déjà amorcé mais combien difficile, d'une publicité canadienne-française.

Toutefois, quelle que soit la rapidité de ce développement, quelle que soit son ampleur, il n'en demeurera pas moins que, par suite de la position géographique du marché québécois et de sa dépendance économique, la majeure partie des documents publicitaires continueront, dans les années qui viennent, à être adaptés de l'anglais.

Il existe déjà des agences canadiennes-françaises et le mouvement vers la création en français se dessine largement dans de nombreuses grandes agences et chez d'importants annonceurs anglophones, mais ce n'est encore qu'une infime partie de la publicité qui est créée en français et nombre d'agences ne pratiquent encore que l'adaptation lorsqu'il s'agit d'annoncer dans notre langue. De plus, une masse énorme de documents publicitaires et commerciaux - imprimés de 
publicité directe, catalogues, inscriptions sur les emballages - continueront sans aucun doute à être rédigés en anglais.

Dans ces conditions, cherchons à améliorer autant que possible la qualité des adaptations, ce qui n'empêchera pas de développer la création et de la soigner. Adaptateurs et créateurs n'œuvrent-ils pas dans le même sens? Les adaptateurs pouvant d'ailleurs devenir d'excellents créateurs, car ils doivent en avoir toutes les qualités.

Puisqu'on devra compter sur les adaptateurs pendant de nombreuses années encore, et compte tenu de la place occupée par la publicité dans notre société, il est inutile d'insister sur le rôle qui incombe à ceux qu'on pourrait appeler les mal-aimés du monde publicitaire.

Mal-aimés, oui, car ils sont généralement les seuls responsables connus du long processus qui amène sous les yeux ou aux oreilles du public ces textes qui le choquent si souvent. Chargés d'une tâche ingrate, complices évidents du système « English creation-adaptation française », les traducteurs servent souvent de boucs émissaires. Pourtant, ils ne sont fréquemment eux-mêmes que les victimes du système et devraient bénéficier de nombreuses circonstances atténuantes.

Bien sûr, une grande part de la responsabilité d'avoir abâtardi la publicité de langue française au Canada incombe à des «traducteurs», en fait à des gens pour la plupart pleins de bonne volonté, mais qui n'ont de traducteur ou d'adaptateur que le nom. Ainsi, quelque imparfait que puisse être le système actuel, ce n'est donc pas tant au principe même de l'adaptation qu'il faudrait s'en prendre, mais plutôt à la qualité des adaptations et par conséquent des adaptateurs. C'est là que se trouve la cause principale de la médiocrité des textes publicitaires français au Canada. C'est là sans doute que se trouve la réforme la plus urgente à faire, avec celle qui consisterait à apprendre le français à des concepteurs que la méconnaissance de la langue oblige à s'exprimer en «joual»sous le prétexte fallacieux de « faire québécois ».

\section{UNE PUBLICITÉ QUI PORTE ET QUI RAPPORTE}

L'adaptation d'un texte publicitaire est une opération toujours ardue, parfois impossible et assez souvent peu recommandable. De ce fait, elle demande à l'adaptateur nombre de qualités et de connaissances, de même qu'une solide expérience. La réussite dans ce domaine n'est pourtant pas impossible. Il ne manque pas d'exemples de campagnes publicitaires fondées sur des adaptations de bonne qualité, supérieures en tout cas à bien des annonces conçues et rédigées en français. Dans l'immense majorité des cas, les lecteurs ou les auditeurs n'eurent pas conscience qu'il s'agissait de textes adaptés et, critère primordial en publicité, le rendement de ces campagnes fut satisfaisant. Certains y verront la preuve de l'assimilation du public canadien-français ; d'autres penseront qu'on avait su donner un esprit vraiment français, ou québécois, à cette publicité.

Mais le mot clé vient d'être dit : le rendement. Le but de la publicité n'est-il pas de convaincre, de vendre ? C'est sans doute énoncer là un truisme, mais on a parfois tendance à oublier des vérités évidentes. Pour l'annonceur, en effet, qu'il soit francophone ou anglophone, il n'est d'autre loi que celle de l'efficacité ${ }^{1}$.

1. Bien sûr, la recherche, parfois forcenée, du rendement à tout prix et par tous les moyens a de quoi choquer, et la publicité fait l'objet à ce propos de critiques qui ne sont pas 
Tout l'art de l'adaptateur consistera donc à produire un document imprimé, sonore ou audio-visuel, qui porte et qui rapporte. Comment?

Il n'est certes pas question de présenter ici un traité, même abrégé, de conception et de rédaction publicitaires. Le sujet a été excellemment traité par des spécialistes et on pourra à ce propos consulter les ouvrages cités dans la bibliographie présentée dans ces pages.

Il est toutefois bon, avant d'aborder les problèmes particuliers à l'adaptation, de rappeler les fondements de toute publicité et quelques-uns des principes qui guident le concepteur et le rédacteur d'un texte publicitaire, dans quelque langue que ce soit.

Les publicitaires et adaptateurs chevronnés ne trouveront certes là rien qu'ils ne sachent déjà et constateront sans doute qu'il est bien difficile de schématiser en quelques paragraphes un sujet aussi complexe. Ces notions sont simplement destinées aux débutants dans l'art difficile de l'adaptation publicitaire et aux traducteurs qui, pour être étrangers à la publicité, n'en «risquent» pas moins d'avoir un jour à adapter une annonce, un prospectus, une lettre de vente, au hasard des travaux qu'on leur confie. Le traducteur ne doit-il pas être le «spécialiste de tout $»$ ?

Tout d'abord, qu'est-ce que la publicité ? Tout le monde le sait, mais il n'est pas inutile d'en rappeler la définition. En voici deux parmi de nombreuses autres.

Celle du Grand Larousse encyclopédique, très générale : «Ensemble des moyens employés pour faire connaître une entreprise commerciale, industrielle, etc., pour faciliter la diffusion de denrées ou marchandises diverses ${ }^{2}$. » (On pourrait ajouter les services.) Et celle de Robert : « Le fait, l'art d'exercer une action psychologique sur le public à des fins commerciales ${ }^{3}$. »

Ces deux définitions se complètent et on remarquera que la dernière fait intervenir la notion très importante d'action psychologique.

\section{CONNAÎTRE LE MARCHÉ, CONNAÎTRE LE PRODUIT}

La publicité étant ainsi sommairement définie, une question se pose. Où se situe-t-elle dans la longue suite des opérations qui mènent de la conception d'un produit à sa vente ?

La définition suivante du marketing - ou de la commercialisation, selon les recommandations faites à la dernière biennale de la langue française - répond à cette question : "Ensemble des activités systématiquement étudiées et coordonnées qui concourent à diriger les produits et les services vers leur marché, en vue d'en assurer la vente. Le marketing inclut notamment l'étude du marché, la présentation, le stockage, la distribution, la promotion des ventes, la publicité ${ }^{4}$. »

La publicité s'intègre donc à un plan général de commercialisation dont elle ne peut être dissociée et que R. E. Oliver décrit ainsi dans son ouvrage Advertising $^{5}$ : «A marketing plan sets out clearly for everyone concerned just what

sans fondement. D'autre part, beaucoup se demandent si la satisfaction du dieu rendement est vraiment incompatible avec le droit d'un peuple à l'" autodétermination publicitaire ", pour adapter une formule qui a beaucoup servi en d'autres circonstances.

2. Grand Larousse encyclopédique, 10 vol., Paris, Larousse, 1960, au mot publicité.

3. Paul Robert, Petit Robert, Paris, Société du Nouveau Littré, 1968, au mot publicité.

4. P. Harbin, Vocabulaire de la publicité, Lagny, Editions de la Gourdine, 1964.

5. R. E. Oliver, Advertising, Toronto, McGraw-Hill Company of Canada, 1969. 
the company plans to sell (product or service); to whom the company plans to sell it (the market); what its advantages are from the customer's point of view (product appeal); how many sales are expected in the next year (sales objective); and how the company plans to sell it (advertising, selling and promotion). 》

De tous ces facteurs essentiels pour le publicitaire, nous ne retiendrons que ceux-ci : le marché et les avantages du produit. Ce sont les plus importants pour les besoins de cette étude.

Le marché se compose de l'ensemble des consommateurs d'un produit ou d'un service. Il convient de bien connaître ces consommateurs. Parmi eux se recruteront les acheteurs éventuels du produit ou du service proposé par l'annonceur. Il est indispensable de savoir où ils se trouvent; qui ils sont (on les classe par sexe, âge, degré d'instruction) ; quels sont leurs goûts, leurs opinions ; quelles sont leurs habitudes d'achat et d'usage du produit en particulier; quels sont les mobiles de leur comportement.

On déterminera aussi les avantages que présente le produit : Répond-il à l'attente du consommateur? Présente-t-il des avantages pour lui ? Pourquoi est-il préféré à un produit concurrent, ou l'inverse ?

Ces renseignements seront obtenus par la recherche - études du marché, sondages d'opinions, études de motivations - ou au moins par une analyse plus ou moins poussée de la chose à vendre et de la clientèle à laquelle elle est destinée. Ils serviront de base à l'établissement du plan de campagne et, entre autres, à déterminer la clientèle qu'on se propose d'atteindre et les arguments qu'on fera valoir auprès d'elle.

En possession de ces données, le concepteur-rédacteur va pouvoir se mettre à l'œuvre.

\section{INFLUENCER L'ACHETEUR}

Son obiectif va être d'inciter le prospecté ${ }^{6}$ à accomplir une action, la plupart du temps, l'acte d'achat. À cette fin, il mettra en ouvre des principes éprouvés, fondés sur la psychologie appliquée et résumés par la formule magique du publicitaire : AIDA - A : attirer l'attention; I : susciter l'intérêt ; D : éveiller le désir; A : provoquer l'achat. Toutes les annonces sont conçues selon ce plan, dans quelque langue que ce soit.

Dans le cas d'un imprimé publicitaire, et de l'annonce en particulier, on peut attirer l'attention visuellement - par l'illustration, la couleur, la valeur esthétique, la disposition typographique, etc. - mais aussi, ce qui nous intéresse particulièrement, par des mots. Le titre, nous le verrons, joue à ce point de vue un rôle primordial.

6. Nous préférerons ce mot à l'anglais prospect pourtant largement utilisé dans les textes de publicitaires français. P. Herbin, dans son Vocabulaire de la publicité, définit ainsi le prospect : « Mot à mot : client en vue. Personne visée comme cible et dont on pense obtenir la décision d'achat en la prenant pour destinataire d'un message de publicité. » Il définit ensuite prospection : " D'une part, recherche de prospects. D'autre part, actions méthodiques sur des clients en vue par envoi de messages publicitaires. " Il semble donc tout à fait normal de dire que les personnes faisant l'objet de la prospection sont des prospectés. Le mot n'est d'ailleurs pas nouveau. On le trouve déjà dans le Traité complet de publicité, de F. W. Crosse, ouvrage probablement traduit de l'anglais (Paris, Dunod, 1939). 
Attirer l'attention est relativement facile. Il est plus difficile de la retenir, surtout dans une ambiance où lecteurs et auditeurs sont constamment sollicités par d'autres sons et spectacles, voire par d'autres messages publicitaires. Le message exprimé devra donc intéresser suffisamment le prospecté pour éviter qu'il s'en détache.

Divers procédés seront alors employés à cet effet, portant sur le fond de la proposition faite au prospecté (caractéristiques de la chose, services rendus, etc.) et sur sa forme, c'est-à-dire la manière dont ces éléments sont portés à sa connaissance (avec l'appui d'une illustration, d'un témoignage d'usager; sous la forme affirmative, interrogative, etc.).

Toutefois, deux conditions importantes sont à observer, auxquelles l'adaptateur devra particulièrement prendre garde. Les procédés devront premièrement, nous dit F. W. Crosse, «être appropriés à la chose à vendre. Pour cela, il faut qu'il existe entre le procédé et la chose un lien quelconque, un rapport de faits ou d'idées, de façon que la chose bśnéficie tout naturellement de l'intérêt suscité. [...] Deuxièmement, être adaptés à la mentalité de la clientèle prospectée ?.» Recommandation qui revêt une importance toute particulière pour l'adaptateur, puisque la clientèle visée par l'annonce qu'il produira peut, à certains points de vue, différer du tout au tout de celle visée par l'annonce en langue originale.

C'est aux mobiles d'acquisition, autrement dit aux instincts, sentiments, tendances du prospecté qu'on fera appel pour susciter le désir de posséder la chose offerte. Ces mobiles se rapportent non pas au produit lui-même, mais aux services qu'on en attend. Autrement dit, le consommateur ne recherche pas un objet, mais les avantages qu'il en tire et grâce auxquels il peut satisfaire un besoin ou une aspiration. Voilà où se situe l'essentiel de l'action psychologique dont il est question dans la définition.

Ainsi, on fera appel au gô̂t de l'économie, au souci du confort, à la tendance au moindre effort, à des satisfactions sensorielles, à l'instinct d'imitation, etc., pour susciter le désir d'acquisition.

On se souviendra toutefois que les mobiles choisis devront être en parfait accord avec les goûts et les habitudes des destinataires du message publicitaire. Autre recommandation qui complète celle donnée ci-dessus et qui, pour des raisons faciles à comprendre, revêt une importance particulière pour l'adaptateur.

$\mathrm{n}$ ne reste plus alors qu'à inciter l'acheteur en puissance à échanger le plus rapidement possible son argent contre le produit annoncé. Comme l'homme est changeant par nature et qu'il a tendance à temporiser, on l'incitera à agir sans tarder en se rendant « dès aujourd'hui » dans un magasin, en envoyant un coupon, etc.

Sur la base de cette argumentation soigneusement mise au point, le concepteur rédigera un texte aussi convaincant qu'il le pourra : simple, concis, de compréhension facile, dans un ton qui pourra être badin ou sérieux, populaire ou élégant, familier ou recherché, selon le public visé ou l'effet voulu. La fin de son travail marquera le début des peines de l'adaptateur.

7. F. W. Crosse, op. cit. 


\section{L'ADAPTATEUR AU TRAVAIL}

Pourtant, pensera-t-on sans doute, ce dernier va avoir la tâche facile. Ne lui apporte-t-on pas un travail tout mâché qu'il ne lui reste plus qu'à transposer dans sa langue maternelle?

La réalité est tout autre. L'adaptateur intervient certes au moment où de nombreux problèmes ont été résolus pour lui, ou du moins semblent l'être.

Un examen sommaire de l'annonce - pour ne prendre que le cas typique de ce moyen de publicité - et des données qui l'accompagnent lui révélera, bien sûr, la nature du produit, le mode de vente, la nature de la clientèle, le nom de la publication (donc le genre de public auquel il s'adresse), le format, etc.

L'adaptateur travaille donc sur un «produit fini 》, dans la préparation duquel tous les éléments importants auront déjà été pris en considération : les arguments de vente, le style général de l'annonce auront été choisis ; une disposition typographique aura été déterminée. En un mot, tout le travail créateur aura été fait. Pourtant, loin de libérer l'adaptateur, ce travail ne lui impose souvent que de nouvelles contraintes. Cette argumentation, ce style, cette disposition ne sont qu'autant de carcans qu'on lui passe.

Le voici dans la situation paradoxale qui consiste à convaincre au moyen d'arguments auxquels les lecteurs sont peut-être insensibles, voire hostiles, ordonnés selon une logique qui n'est pas la leur, exprimés en faisant appel à des images, des métaphores, des jeux de mots sans équivalents dans leur langue maternelle, ou rappelant des habitudes de vie qui leur sont étrangères, le tout dans une disposition typographique souvent fondée, à un caractère près, sur l'espace disponible pour un titre, sur un nom de marque, un graphisme... Situation de tout repos en vérité ! dont l'adaptateur ne pourra triompher qu'en suivant la même démarche que son collègue, le concepteur-rédacteur.

Comme lui, il devra bien connaître le produit. En traduction, on sait combien il est important de posséder le sujet. En adaptation publicitaire, cette connaissance est primordiale. En effet, comment pourrait-on autrement présenter un produit, le décrire, en faire valoir les qualités ? Connaître parfaitement la chose à vendre, voilà la première recommandation donnée aux vendeurs. L'adaptateur publicitaire n'est autre chose qu'un vendeur.

Pourtant, les renseignements donnés à l'adaptateur, même travaillant au sein d'une agence, sont souvent fragmentaires. Il devra donc s'efforcer d'obtenir tous les documents pouvant lui apporter les renseignements nécessaires à l'élaboration de son texte, en particulier lorsque la présentation du produit implique des données techniques. Idéalement, il devrait pouvoir examiner le produit, l'appareil, la machine, l'automobile qu'il va « vendre ». Il devrait avoir l'occasion de rencontrer ceux qui vendent réllement ce produit et sont en relation directe avec le public. De ces contacts naissent souvent des idées fructueuses, des façons d'envisager la mise en valeur de la chose à vendre, des arguments insoupçonnés, des tournures même qu'on emploiera avec profit.

Il serait de même souhaitable que l'adaptateur puisse prendre connaissance, sinon des études et sondages qui ont mené au choix de l'axe de la campagne, mais du moins des documents qui les résument et à l'aide desquels le concepteur a travaillé, en particulier l'argumentaire (le rationale des Américains). L'adaptateur 
pourra ainsi connaître les arguments qu'on veut développer et l'approche qu'on veut adopter, lesquels ressortent sans doute à la lecture de l'annonce, mais pas toujours clairement. Aussi, est-il bon d'en avoir la confirmation et de connaître la raison de leur choix. D'autre part, ces documents apprendront si les études, sondages et analyses ont été faits auprès des consommateurs francophones auxquels on va s'adresser ou bien, comme c'est encore souvent le cas au Canada, surtout quand il ne s'agit pas de produits de grande consommation, sur un marché uniquement anglophone... l'annonceur se fiant à l'extrapolation des données qu'il a recueillies pour toucher juste. Espoir souvent vain... et qui fera porter à l'adaptateur la lourde responsabilité de «corriger le tir». Ce qu'il devra faire, presque toujours, intuitivement et grâce à sa connaissance de «son » marché et de «ses» consommateurs.

\section{QU'EST-CE QU'UN CONSOMMATEUR ?}

Pourtant on sait combien le consommateur est, quoi qu'on en dise, difficile à influencer. En voici d'ailleurs un excellent portrait, tiré justement d'une annonce. (L'illustration représente une femme faisant son marché, et symbolisant le consommateur.)

Chez nous, c'est elle qui dirige la création.

Elle a tous les défauts. Elle est tête en l'air, versatile : pour un rien elle abandonne la lecture d'une annonce.

Elle est distraite : quelquefois, elle ne voit même pas les films que nous lui soumettons. Elle est irascible : certaines couleurs, certains mots mal utilisés la mettent hors d'ellemême.

Elle se permet de tout juger en quelques instants et elle ne fait jamais d'efforts pour comprendre.

[...] notre juge suprême en matière de création, c'est le consommateur 8 .

Il suffit simplement de remplacer dans cette annonce le mot «création» par le mot « adaptation ». Pour le reste, tout est dit sur les « défauts » du consommateur.

De cette description, retenons simplement un point pour l'instant : « Il suffit de certains mots mal utilisés pour indisposer le consommateur. » Et ajoutons, en pensant au consommateur soumis à une publicité mal adaptée - ou inadaptée, si l'on préfère : certains arguments ne le touchent pas, certaines situations où il ne se reconnaît pas l'agacent, certains sentiments le choquent.

Or, la publicité c'est aussi savoir plaire. Plaire, c'est avant tout ne pas choquer. Et comment ne pas choquer si l'on ne connaît pas tout ce qui différencie le destinataire d'une annonce originale de celui de l'adaptation de cette annonce ?

De là, la nécessité de connaître à fond les prospectés auxquels s'adresse la publicité, même si - pour ne prendre que le cas du Québec - ces acheteurs éventuels ont un mode de vie proche de celui de leurs compatriotes anglophones, même s'ils semblent répondre aux mêmes motivations.

Le texte qui suit est à nouveau celui d'une annonce publiée par une agence de publicité - japonaise cette fois — vantant ses services. Il suffirait d'y changer peu de chose et de remplacer «Japan» par «Québec» pour qu'il puisse être utilisé par une agence canadienne-française offrant ses services sur le marché nord-américain. (L'illustration représente un joueur de baseball.)

8. L'Expansion, octobre 1971, p. 50, annonce de l'agence McCann-Erickson. 
[...] In Japan, baseball is a national passion, just the way it is in the U.S. There are lots of other similarities, too. Television antennas over most roofs, traffic snarls, jampacked department stores, and millions of people who are constantly aspiring to a better life.

But don't let the similarities fool you. Beneath the 20th century surface, Japan's culture is as different from yours as can be. To sell in this rich market, you have to combine an understanding of Japan's different ways and traditions with all the modern marketing techniques ${ }^{9}$.

Nous aurions pu citer les annonces d'agences canadiennes-françaises développant la même argumentation à l'intention des annonceurs anglophones du Canada. Nous avons préféré cet exemple, non seulement pour son exotisme mais parce qu'il nous semble plus frappant.

\section{COMMENT PLAIRE AU CONSOMMATEUR ?}

On imagine facilement en effet que les différences culturelles existant entre Nord-Américains et Japonais sont grandes malgré des analogies de surface. Les différences séparant anglophones et francophones du Canada sont sans doute moins nombreuses et moins marquées. Il ne faut cependant pas en conclure que les deux ethnies se composent de consommateurs semblables en tous points parce que ceux-ci sont également amateurs de hockey et de télévision... comme le font encore trop d'annonceurs anglophones qui, par ailleurs, trouvent tout à fait normal qu'une annonce américaine doive être canadianisée pour pouvoir être publiée sur le marché canadien-anglais. Ces différences dans les façons de vivre (habitudes alimentaires et vestimentaires, par exemple), dans le mode de penser, dans certains réflexes même, ne peuvent paraître que de simples curiosités à l'homme de la rue, mais elles prennent une tout autre importance quand il s'agit de vendre et elles entraînent certainement les plus grandes difficultés de l'adaptation publicitaire.

L'étude comparée des caractéristiques psychologiques, sociologiques et culturelles des consommateurs francophones et anglophones déborde le cadre de cet article. Qu'il suffise de dire en résumé que l'adaptateur devra veiller à éliminer de l'annonce tout ce qui peut choquer le consommateur dans ses croyances, ses sentiments - y compris le sentiment national, — ses traditions, ses attitudes, ses coutumes, son comportement, autrement dit dans tout ce qui constitue son patrimoine culturel. Il devra également effacer tout argument qui pourrait porter à faux et nuire à la crédibilité de l'annonce. En un mot, il devra faire disparaître tout ce que la publicité comporte d'étranger au milieu auquel il s'adresse, en s'identifiant parfaitement à ce milieu, en le connaissant à fond. Il devra posséder aussi une sensibilité et une intuition qui lui permettent de déceler tout ce qui, dans le texte original et dans les autres éléments de l'annonce - l'illustration en particulier - pourrait provoquer une réaction défavorable du prospecté. Il lui appartiendra alors de choisir, et s'il le peut après avoir consulté des spécialistes du marché auquel il s'adresse, l'argument ou l'approche de nature à remplir la même fonction que les éléments indésirables de l'annonce originale qu'il se propose d'éliminer. 9. Dentsu/Japan, annonce citée par J. S. Wright et D. S. Warner, dans Advertising, New
York, McGraw-Hill Book Company, 1966 . 


\section{ET LORSQUE LA PUBLICITÉ EST INADAPTABLE ?}

On rencontrera toutefois des annonces qu'aucun effort d'adaptation ne pourrait rendre acceptable. C'est le cas de l'annonce citée ci-après, qui présente un cas d'inadaptabilité totale due à plusieurs facteurs.

On trouve quotidiennement dans les journaux et revues de langue anglaise nombre d'annonces inadaptables (que l'on a parfois la surprise de voir «adaptées » dans des publications de langue française), toutefois le texte que nous avons choisi est en français, car nous pensons qu'il rend la démonstration plus probante. Il s'agit d'une annonce de Volkswagen, typique de l'humour qu'a su donner à la publicité de cette firme l'agence Doyle, Dane, Bernbach ${ }^{10}$. (L'illustration représente une Volkswagen, vue de l'avant. Le couvercle du coffre est relevé. Devant la voiture, un nombre impressionnant de bouteilles de vin, étiquetées et cachetées.)

En titre :

Le coffre de la nouvelle VW contient maintenant 260 litres.

Ça s'arrose.

Le corps de lannonce :

Et copieusement.

Parce que de 140 à 260 litres ce n'est pas ce qu'on appelle un petit changement.

Et si vous ajoutez à ce nouveau coffre avant les 140 litres du coffre arrière, avouez qu'il y a de quoi se livrer à de sérieuses libations.

Ce n'est pas tout.

En prenant de la bouteille (il y a 25 ans que nous construisons la Coccinelle), nous avons apporté quelques autres améliorations.

Comme la puissance du moteur : $44 \mathrm{CV}$ DIN au lieu de 36.

Comme le nouveau rayon de braquage : 4,5 m au lieu de $5,5 \mathrm{~m}$.

Comme la nouvelle climatisation (partant du principe que certains vins se boivent bien chambrés).

Nous perfectionnons la Coccinelle depuis longtemps.

Mais il aurait été dangereux de sabler le champagne à chaque perfectionnement que nous avons apporté à la Coccinelle.

Depuis la création, il y en a eu plus de 15280.

Attention à l'alcool-test.

On voit que, dans cette annonce, les facteurs d'inadaptabilité sont de plusieurs ordres. Tous interdiraient l'adaptation en anglais, quel que soit le marché ; certains empêcheraient toute possibilité de diffusion, même sur un marché de langue française comme le Québec.

Tout d'abord, toute l'annonce est bâtie sur l'association d'idée entre le mot « litre » et la célébration « vineuse » d'un perfectionnement apporté à la voiture. En effet, en France, la contenance des coffres de voiture s'exprime en litres, alors qu'elle s'exprime en pieds cubes dans les pays utilisant les mesures anglaises, dont le Québec. Ce fait suffirait à interdire l'adaptation. Voyons cependant les autres facteurs.

Deuxièmement, la consommation de vin n'est pas courante dans ces derniers pays et, même si l'on avait la plaisante idée de célébrer le perfectionnement annoncé, ce ne serait probablement pas avec du vin.

Troisièmement, il ne viendrait à aucun annonceur nord-américain l'idée d'associer alcool et automobile en tempérant — sans jeu de mots — l'idée par un

10. Le Nouvel Observateur, 31 mai 1971, p. 7, annonce Volkswagen. 
《attention à l'alcool-test». Ce serait contraire aux règles de l'éthique professionnelle et il est certain que la réaction du public anglo-saxon - peut-être par puritanisme - serait de toute façon très défavorable. Il serait intéressant d'étudier à ce sujet les réactions des Québécois. D'esprit latin, plus attachés à la joie de vivre que leurs compatriotes anglophones, ils seraient sûrement plus sensibles à ce genre d'humour et ne s'en offusqueraient pas.

Quatrièmement, le texte comporte des jeux de mots rattachés à l'enchaînement d'idées « litre-arroser-vin», par exemple : «en prenant de la bouteille», ou fait appel à certaines connaissances que le grand public nord-américain n'a pas : « certains vins se boivent bien chambrés ».

Cette annonce contient ainsi la gamme complète des facteurs d'inadaptabilité, qu'on peut ranger dans trois grandes catégories : psychologiques, culturels, linguistiques. (Nous mettons à part les facteurs de caractère matériel, typographique, entre autres.) De plus, elle démontre qu'au moins les deux premiers facteurs peuvent interdire l'adaptation à l'intérieur même d'une communauté linguistique. (Dans certains cas, des facteurs linguistiques, comme l'emploi d'expressions familières ou argotiques peu répandues, rendraient également inefficaces la diffusion de textes d'un pays de langue française à un autre.)

\section{SAVOIR ADAPTER, C'EST SAVOIR CHOISIR}

On voit également ici que la difficulté d'adaptation des textes augmente en proportion même de leur contenu en éléments des trois catégories citées ci-dessus. Or, de tous les textes publicitaires, l'annonce est le plus chargé de ces éléments, et en particulier l'annonce pour des produits de grande consommation ou de biens de grande diffusion. L'adaptation d'annonces à fort contenu technique ou de textes presque uniquement descriptifs - prospectus, dépliants, catalogues - ne pose que des problèmes mineurs.

En somme, savoir adapter, c'est avant tout savoir choisir. Lorsque l'adaptateur aura su faire son travail d'élimination, il sera en mesure de produire un texte qui pourra paraître conçu en français, et qu'on n'accusera pas d'angliciser le lecteur, car il aura été repensé... mais à condition toutefois que l'expression soit vraiment française. C'est là que nous abordons l'aspect proprement linguistique du travail de l'adaptateur, ce publicitaire qui, tout compte fait, doit être concepteur, rédacteur et traducteur tout à la fois.

L'adaptation est diversement définie par les dictionnaires ${ }^{11}$. Le Grand Larousse encyclopédique en donne une définition très générale : "Arrangement d'une œuvre étrangère autrement qu'en sa traduction littérale ${ }^{12}$. 》Robert limite l'acception du terme : "Traduction très libre d'une pièce de théâtre, comportant des modifications nombreuses qui la mettent au goût du jour ou la rajeunissent ${ }^{13}$.»

11. Nous nous en tiendrons au sens large du mot, et non au sens spécialisé qu'en donnent Vinay et Darbelnet pour décrire un des procédés de traduction : « Utilisation d'une équivalence reconnue entre deux situations » (J.-P. Vinay et J. Darbelnet, Stylistique comparée du français et de l'anglais, Paris, Didier, 1958, p. 4). L'adaptation, dans cette acception, n'est donc qu'un des procédés auquel le traducteur publicitaire fera appel, et même fréquemment. J.-P. Vinay écrit d'ailleurs : "La publicité est un des domaines privilégiés de l'adaptation " (J.-P. Vinay, "La traduction humaine", dans le Langage, Encyclopédie de la Pléiade, Paris, Gallimard, 1968, p. 750).

12. Grand Larousse encyclopédique, au mot adaptation.

13. Paul Robert, Petit Robert, au mot adaptation. 
C'est dans le Dictionnaire encyclopédique Quillet qu'on trouve une définition s'appliquant presque parfaitement au travail de l'adaptateur publicitaire : «Travail littéraire par lequel un écrivain, prenant pour texte l'ouvre d'un autre auteur en langue étrangère, la transforme en une production analogue qu'il croit plus appropriée au goût de ses compatriotes ${ }^{14}$. »

Nous espérons que les auteurs de ce dictionnaire ne nous en voudrons pas d'emprunter cette définition et de la modifier quelque peu pour décrire ainsi la tâche de l'adaptateur publicitaire : «Travail par lequel un traducteur transforme un texte publicitaire en langue étrangère en une production analogue qu'il estimera apte à remplir auprès des consommateurs de sa langue la fonction à laquelle le texte original était destiné. »

\section{LES « BELLES EFFICACES 》}

L'adaptation consistera ainsi à écrire sur la trame suggérée par l'annonce originale un nouveau texte répondant aux exigences que nous avons citées. Là, il ne sera pas question de respecter scrupuleusement la pensée de l'auteur, ni même son style. Il s'agira plutôt d'atteindre le but recherché avec l'annonce originale, et la voie pour rejoindre ce but pourra s'écarter sensiblement de celle suivie par le concepteur. L'adaptateur pourra donc présenter sans rougir une de ces belles infidèles tant décriées dans d'autres domaines. Ainsi libéré, il aura la partie facile, pensera-t-on. Pas tellement car, plus qu'une belle infidèle, son adaptation devra être une « belle efficace ».

Dans sa préface aux Problèmes théoriques de la traduction ${ }^{15}$, Dominique Aury écrit à propos des traducteurs : "Nous ne sommes même pas sûrs de nous entendre entre nous : les «techniques », comme nous disons dans notre jargon, envient les «littéraires», parce que les littéraires n'ont pas de difficultés de vocabulaire, et les littéraires envient les techniques, parce que les techniques n'ont que des difficultés de vocabulaire. »

Nous pourrions ajouter : «Les «publicitaires» envient les « techniques» et les «littéraires»car outre les difficultés de leurs collègues, ils connaissent le souci du rendement de leur texte. »

Nous revenons ainsi au mot clé de la publicité : le rendement. Comment, après avoir choisi les éléments susceptibles de persuader le prospecté, l'adaptateur va-t-il les mettre en cuvre pour obtenir le maximum d'efficacité ? Ou, pour poser autrement la question, comment, après avoir réglé le problème du fond, va-t-il résoudre celui de la forme? Avant d'envisager ce problème sous l'angle des rapports avec la langue de départ, il est bon de considérer d'une façon générale l'outil dont l'adaptateur va se servir : la langue publicitaire.

Un publicitaire a dit : «La publicité est une littérature de combat. » La formule est frappante et exacte. Accrocher le lecteur, l'intéresser, le persuader, c'est mener une lutte contre d'autres sujets d'intérêt, contre l'indifférence, contre des réactions défavorables, conscientes ou inconscientes. Une lutte dans laquelle la langue, véhicule de communication, joue un rôle de premier plan.

Comment décrire cette langue publicitaire? P. Herbin en esquisse les traits

14. Dictionnaire encyclopédique Quillet, 6 vol., Paris, Librairie Aristide Quillet, 1958, au mot adaptation.

15. G. Mounin, les Problèmes théoriques de la traduction, Paris, Gallimard, 1963, p. vII. 
principaux, indirectement il est vrai, mais excellemment, dans son ouvrage Jeux de mots ${ }^{16}$, à propos de l'emploi du «franglais » et de la formation des rédacteurs publicitaires :

Ainsi, de futurs publicitaires seront moins tentés de recourir à la solution paresseuse du franglais 17 si on leur a inculqué la hantise d'être compris de tous, si on leur a donné le goût du bien écrire, si on leur a fait une langue riche, saine, savoureuse, musclée, débrouillarde, si on leur a montré que pour vendre des tissus ou des détergents, le français était fort capable de leur fournir la sève d'un vocabulaire authentique, servi par la rigoureuse souplesse d'une syntaxe irréprochable.

Retenons le premier point : «être compris de tous ». C'est de toute évidence la condition essentielle pour persuader, mais on l'oublie si souvent! Être compris de tous par l'emploi d'un vocabulaire simple, précis, correct. Cette dernière épithète prend une importance particulière au Québec où se posent des problèmes de compréhension de certains termes, en particulier de termes techniques. En fait, ce ne sont que de faux problèmes. En effet, le terme juste est compris dans l'immense majorité des cas à condition, bien sûr, qu'il ne soit pas trop savant, ni trop nouveau, auquel cas il sera facile d'en fournir discrètement le sens.

\section{SAVOIR COMMUNIQUER}

Le principal problème de vocabulaire qui se posera au traducteur sera l'appellation de produits, appareils, services ou concepts nouveaux. On sait que nous avons souvent au Québec le privilège d'être les premiers à devoir baptiser ces nouveaux-nés. Il faudra procéder avec le plus grand soin, car l'appellation choisie, même si elle est mauvaise, risque de se fixer et de passer dans le vocabulaire courant.

Ce problème peut être purement technique, comme dans le cas de power train $^{18}$ dont la traduction fautive continue pourtant d'être employée (en publicité, il est difficile de faire machine arrière, même pour corriger une erreur). Il peut se compliquer du fait de la fonction publicitaire de l'appellation nouvelle, qui est souvent difficile à concilier avec les exigences linguistiques, ou même le simple bon sens.

En voici un exemple entre mille autres. Il y a quelques années, une société horlogère américaine a lancé la première montre à diapason. Voulant clairement indiquer que son invention était autre chose qu'une montre, plus qu'une montre, le mot watch avait été banni de la publicité et remplacé par timepiece, qui ne possède pas d'équivalent en français. Un malheureux publicitaire se vit ainsi forcé d'utiliser un néologisme - horomètre — bien formé, mais d'emploi assez difficile. (Essayez de placer dans la conversation : «Avez-vous vu mon horomètre-bracelet ? ») Les horomètres étaient, paraît-il, particulièrement robustes, mais le mot, lui, n'a pas résisté à l'usage... où n'y est pas entré.

Tous les publicitaires canadiens-français savent qu'il est particulièrement dif-

16. P. Herbin, Jeux de mots, Lagny, Editions de la Gourdine, 1967, p. 21.

17. Remplaçons "franglais " par " joual " et les remarques qui suivent gardent toute leur valeur.

18. Citons la "traduction" pour le moins cocasse de power train, ou groupe moto-propulseur d'une automobile, donnée à la hâte par un constructeur à l'occasion de l'annonce d'une nouvelle garantie : "rouage d'entraînement ». Dans ce cas, la chose n'était certes pas nouvelle, mais le terme jusque-là strictement réservé au domaine technique faisait irruption dans le domaine public, ce qui revient à une création. 
ficile de faire comprendre à un annonceur anglophone que si l'on peut facilement donner en anglais une appellation nouvelle à un produit, souvent forgée de toutes pièces, cela est beaucoup plus difficile en français et souvent d'ailleurs peu recommandable.

Donc, «vocabulaire authentique », mais aussi « rigoureuse souplesse d'une syntaxe irréprochable ». Autre recommandation qui est loin d'être inutile, et à laquelle les résultats d'un sondage effectué par l'Office de la langue française sur la qualité du français dans la publicité donne, s'il en était besoin, encore plus de poids :

Il a été établi de façon: significative qu'un passage en français correct est mieux compris par le public en général que s'il est rédigé en français négligé ;

La population, en général, désire que l'on emploie un français de bonne tenue et se rend compte des déficiences d'un message qui lui est présenté en une langue incorrecte ; La population est naturellement prédisposée en faveur d'une langue familière mais correcte et elle appréhende les anglicismes de toute sorte ;

Les personnes très instruites peuvent beaucoup plus facilement que les personnes peu instruites comprendre les messages mal rédigés ou mal traduits. Cela ne veut pas dire qu'elles les apprécient. Un message mal conçu ne sera compris que par une minorité alors qu'il prétend s'adresser à l'ensemble de la population ;

Une annonce en français correct, tant sur le plan du vocabulaire que de la syntaxe, correspondant au français international, mis à part certains régionalismes acceptés ou de bon aloi, est aussi bien comprise qu'une annonce joualisante et sera beaucoup plus sûrement assimilée par le plus grand nombre qu'une annonce mal traduite 19 .

Le rédacteur publicitaire, donc l'adaptateur, devra aussi posséder le « goût du bien écrire » et une « langue riche, saine, savoureuse, musclée ».

\section{DE LA RÉDACTION, PLUS QUE DE LA TRADUCTION}

Recommandation qui n'est pas non plus sans utilité. La grande majorité des traductions publicitaires que nous lisons ou entendons quotidiennement ne sontelles pas précisément simplement traduites, et mal traduites, alors qu'elles devraient être rédigées - et bien rédigées - en français. «Bien écrire», l'expression a un relent suspect pour beaucoup de gens, et en particulier pour beaucoup de publicitaires. Pour eux, bien écrire, c'est faire des phrases, c'est s'exprimer dans une langue littéraire, recherchée, ampoulée, une langue de sermon. Pour le publicitaire, bien écrire, ce sera simplement s'exprimer dans une langue correcte mais vivante, simple mais colorée, en somme située à l'opposé de celle de nombreux textes publicitaires encore actuellement publiés (et parmi eux, mais de moins en moins il est vrai, des textes préparés par des agences).

Certes l'adaptateur publicitaire travaille souvent dans des conditions difficiles. On lui donne la plupart du temps des délais d'exécution insuffisants. Il doit d'autre part compter avec l'espace ou le temps qu'on lui alloue pour la diffusion de son message. Toutefois, ce ne sont pas là des excuses à la médiocrité, sinon à l'incorrection des textes.

Les annonces adaptées souffrent d'une manière générale de plusieurs défauts. D'abord, elles sentent la traduction. Le traducteur n'a pas su se libérer de l'anglais. D'autre part, et sans doute par conséquence directe du défaut précédent, elles sont ternes et sans vie. Le style est lourd, alors qu'il devrait être alerte et pétillant,

19. Le Devoir, 2 novembre 1971 , p. 16. 
d'autant plus que le ton plaisant est de plus en plus employé en publicité. Il n'y a rien de plus pénible à lire (quand on se force à lire) qu'une annonce conçue sur ce ton badin maintenant à la mode et qui a été traduite laborieusement et pesamment. Que l'adaptateur n'oublie jamais que la traduction doit être l'expression la plus naturelle du message de la langue de départ. Ce qui veut dire un message totalement affranchi des contraintes de la forme, dans une langue concrète, facilement intelligible et le plus souvent dans le registre d'expression de la langue parlée.

Possédant les bases indiquées ci-dessus, auxquelles il ajoutera évidemment une bonne connaissance de la langue de départ, l'adaptateur publicitaire devra sans cesse travailler son style. Il devra lire, écouter, retenir, en particulier les formules vivantes et familières, nées spontanément dans la conversation, et qui, employées dans un texte, donneront ce tour naturel, le ton juste qui relancera l'intérêt, fera retenir un argument, l'expression qui donnera le slogan frappant. L'adaptateur devra rédiger aussi avec la plus grande économie de moyen. En effet, le temps et l'espace étant comptés, la publicité, comme le journalisme, oblige à la concision. En ce sens également, c'est une excellente école de style et, à ce titre, les exercices d'adaptation publicitaire devraient faire partie du programme de formation de tous les traducteurs ${ }^{20}$.

Alexandre Vialatte, à la fois romancier, traducteur et journaliste, fait à propos de la traduction littéraire une observation qui s'applique parfaitement à l'adaptation publicitaire : "J'aime la contrainte qui oblige à la précision et à l'ellipse. D'ailleurs, ce qui est intéressant chez un auteur, ce qui enchante, c'est le «ton», qui peut aussi bien se trouver dans un article de journal. Le grand art, c'est de donner beaucoup en quinze lignes ${ }^{21}$. »

Quinze lignes, souvent l'adaptateur ne dispose pas de beaucoup plus pour «donner le ton», pour faire passer le maximum avec un minimum de mots.

\section{SUR TOUS LES TONS}

Arrêtons-nous un instant à cette question du ton. En effet, le ton de l'annonce originale peut causer quelques difficultés à l'adaptateur.

Nous avons vu que l'annonce peut être rédigée dans des tons bien différents. Longtemps écrit dans un style très littéraire et sur un ton emphatique ${ }^{22}$, le texte publicitaire a considérablement évolué. Les exagérations émoussant la crédibilité, les publicitaires ont donc adopté un ton qui les rapprochent du public qu'ils visent. Leur registre pourra aller du ton neutre de la description d'une machine au ton badin, familier, humoristique même, des annonces pour les produits les plus variés, en passant par le ton littéraire, presque noble, de la publicité de prestige ou de notoriété ${ }^{23}$.

20. C'est d'ailleurs le cas à l'Université de Montréal où le programme de troisième année du baccalauréat en traduction comprend des travaux pratiques d'adaptation publicitaire.

21. Le Monde, supplément au numéro 7833,21 mars 1970 , p. Iv.

22. "Nous entrons đans la période de la science, il faut un air doctoral, un ton d'autorité pour s'imposer au public », fait dire Balzac au rédacteur du prospectus en faveur de l'huile céphalique; conseil qui semble avoir été suivi longtemps après l'époque de lauteur de la Comédie humaine (César Biroteau, Paris, Gallimard, "Bibliothèque de la Pléiade ", 1952, p. 440ss.).

23. Expression qui, comme tant d'autres en publicité, disparaît au profit d'un calque de 
Le premier cas ne pose aucun problème. Le ton badin exige, nous l'avons vu également, un style alerte. Le ton familier demandera surtout de la part de l'adaptateur une parfaite maitrise dans le choix du vocabulaire et des tours, en fonction des niveaux de langue. Familier ne veut pas dire vulgaire. Dans certains cas aussi, la familiarité - registre courant en nord-américain - ne sera nullement de mise dans l'annonce en français. Quant au ton littéraire de la publicité de prestige, il devra être la plupart du temps légèrement modéré en tenant surtout compte du fait que l'annonce nord-américaine de ce type a fréquemment tendance à tomber dans le pompiérisme. C'est toutefois dans le cas des annonces humoristiques que le ton juste sera le plus difficile à obtenir... du moins dans les rares cas où l'annonce sera adaptable, car ce qui fait rire un anglophone ne fait pas forcément rire un francophone.

En toute circonstance, ce difficile problème ne pourra être résolu que grâce à une qualité que doit posséder, et particulièrement cultiver, l'adaptateur publicitaire : le goût ${ }^{24}$.

On voit souvent en effet des adaptations irréprochables au point de vue de la technique publicitaire et de la langue, mais dont le ton, la manière, ne sont pas ceux d'une annonce qui aurait été rédigée en français. Le principal écueil de l'adaptation publicitaire est là : comment faire disparaître ces traces, cet accent indéfinissable qui trahissent l'origine étrangère ?

Il nous reste maintenant à envisager quelques problèmes concrets qui se posent à l'adaptateur de textes publicitaires, et particulièrement d'annonces. Il s'agit en fait de difficultés touchant plus la pratique que la théorie. L'adaptateur possédant déjà — ou devant posséder - une formation de traducteur, n'éprouvera aucune difficulté à saisir le sens du message en langue de départ, ni à lui donner dans sa langue une forme correcte. Mais ce n'est pas suffisant, puisqu'il devra, ne l'oublions pas, produire un texte qui «porte et qui rapporte», et cela en respectant de nombreuses servitudes matérielles.

Imaginons donc l'adaptateur aux prises avec une annonce. Peu importe son style, peu importe la chose à vendre; nous nous en tiendrons aux difficultés générales, communes à tous les textes de ce genre.

La lecture du texte, complétée par l'examen de l'illustration et de quelques autres documents, aura déjà permis de répondre aux grandes questions que se pose l'adaptateur : Que vend-on? Comment? À qui ? Où? Dans quel espace? Il pourra ainsi décider de la démarche générale et de l'approche qu'il devra adopter.

\section{"LE COMMENCEMENT EST PLUS QUE LA MOITIÉ DU TOUT *}

Le premier élément de l'annonce avec lequel l'adaptateur viendra en contact sera évidemment le titre. C'est le plus important et, sans aucun doute, le plus difficile à traiter. Du titre dépend en effet pour une très grande part le rendement de l'annonce. On estime que le titre est lu par cinq fois plus de personnes que le corps de l'annonce. Si le titre intéresse le lecteur, le texte qui suit a un certain nombre de chances d'être lu. Sinon...

24. Même si le mauvais goût est aussi un moyen de se faire remarquer... largement exploité ces derniers temps. 
Le rôle du titre est donc essentiellement d'accrocher, d'attirer l'attention, d'amorcer l'argumentation développée dans l'annonce, d'effectuer la liaison avec l'illustration et le texte.

Définir le rôle du titre, c'est définir ses qualités. Le titre devra avoir au moins l'une de celles-ci : Phrase d'attaque, le titre doit être frappant, intéressant, aussi vivant que possible. On préférera donc les titres dynamiques aux titres statiques. S'adressant à une catégorie particulière de prospectés, le titre doit faire en sorte que ceux-ci se reconnaissent immédiatement comme des acheteurs possibles ; autrement dit, le titre doit être sélectif. Exemple : Le titre «Attention, bientôt l'hiver!» n'a aucune sélectivité. Que vend-on? Des pneus à neige, du sirop pour la toux, des appareils de chauffage?

Devant renseigner, le titre doit exprimer clairement une information, une proposition complète, sans égarer l'intérêt du lecteur sur des détails inutiles. Naturellement, ces buts peuvent être atteints de plusieurs manières et on peut distinguer de nombreux genres de titres. Qu'il suffise de citer les formules les plus courantes :

Affirmer un fait :

Volvos come in

blue, green, white,

yellow and red.

No rust.

Ou encore :

Vous pesez dix ans de trop. [Annonce pour une eau minérale.]

On remarquera que ces deux titres, dans le mode plaisant, jouent sur les mots.

Poser une question :

What makes Wonder Bread so fresh?

Commander une action :

Try a sip of Galliano. [Annonce pour une liqueur.]

Intriguer :

Les Araucans adoraient le tinéo. [Titre complété par une illustration amusante, pour des meubles plaqués en bois de tinéo.]

L'effet est obtenu ici par deux mots inconnus de la grande majorité des lecteurs, qui incitent à trouver une explication dans le texte.

Rien n'interdit d'ailleurs de combiner deux de ces formules. Par exemple poser une question et y répondre par l'énonciation d'un fait :

What sets Shaver apart from the other top breeders?

13 more eggs from every layer housed.

Deux principaux problèmes, parmi bien d'autres, vont se poser dans l'adaptation du titre. Le premier concerne l'espace. En effet, d'une part, l'anglais se prête aux titres courts, percutants; d'autre part, il faut généralement plus de mots en français qu'en anglais - et des mots plus longs - pour exprimer la même idée. Or, comme la maquette de l'annonce a été conçue en fonction du nombre de caractères du titre anglais, l'adaptateur est contraint de respecter, parfois à quelques caractères près, la longueur de ce titre, en même temps que les coupures par lignes, ce qui multiplie les problèmes. S'il rédige un titre trop long, celui-ci devra être composé dans un caractère plus petit, et parfois trop petit, ou encore l'augmentation du nombre de lignes rompra l'équilibre soigneu- 
sement calculé des masses de l'annonce. On aura ainsi une annonce «tassée», insuffisamment aérée, qui ne tirera pas l'œil, ou rebutera le lecteur.

La rédaction d'un titre devient, dans ces conditions, un exercice très difficile. Seule la pratique peut enseigner à trouver la formule frappante et suffisamment concise, à éliminer l'idée non essentielle, quitte à la compenser par ailleurs.

La deuxième difficulté fréquemment rencontrée réside dans l'emploi en langue de départ d'une locution, d'un jeu de mots, d'une allusion à une ceuvre littéraire ou à une coutume qui sont sans équivalent ou dont l'équivalent a une autre connotation dans la langue d'arrivée. Problème qui se complique au point d'interdire toute solution lorsque l'annonce originale associe directement le titre à une illustration.

\section{QUELQUES TITRES PROBLÈMES}

Voici quelques exemples. D'abord, un jeu de mots non directement lié à l'illustration (un paquet de cigarettes) : «Do yourself a flavour ». L'annonce, publiée dans une revue canadienne, l'a peut-être aussi été en français, mais nous ne disposons pas de cette dernière version. Toutefois, il est évident que, dans ce cas, l'adaptation s'est imposée ${ }^{25}$.

Maintenant, deux exemples d'allusion à des œuvres littéraires célèbres. Il s'agit (encore une fois) d'une annonce de Volkswagen. L'illustration représente la silhouette bien connue de la Coccinelle, figurée d'un seul trait de crayon. En titre : «The Shape of Things to Come». L'annonce étant parue dans Life, nous ne pensons pas qu'elle ait été adaptée en français. De toute façon, quel que puisse être le titre français (ou en toute autre langue), il y aura perte d'une allusion à la fois prestigieuse et amusante au titre de l'ouvre de H. G. Wells, maintenant presque devenu locution du langage courant... Laissons le lecteur s'amuser à trouver un titre à cette annonce.

Autre exemple d'allusion à une cuvre connue. Il s'agit cette fois d'une annonce de prestige d'Alitalia publiée dans le programme de l'Opéra du Québec. Le texte est bilingue; l'illustration représente simplement une main de femme tenant des jumelles de théâtre. En titre : «A Tale of two Cities». Le texte développe le thème du rapprochement exercé par l'opéra entre une ville comme Montréal et une ville italienne (qu'on suppose être Milan, capitale mondiale de l'opéra). Solution de l'adaptateur français : «Un lien entre les nations ».

La traduction du titre de l'œuvre de Dickens ne donnerait évidemment pas un titre intéressant mais la rédaction d'un nouveau titre contenant une nouvelle idée devra évidemment entrâner une adaptation parallèle du texte, afin de développer l'idée ainsi introduite. Cette idée s'applique d'ailleurs mieux au rôle joué par une compagnie aérienne. En fait, l'adaptation est ici largement dépassée et nous avons plutôt un cas de création.

Il est intéressant de citer ces deux textes car ils montrent comment, précisément, la seule adaptation d'un titre peut entraîner l'adaptation du texte original... surtout quand celui-ci est plutôt faible :

25. La solution paresseuse adoptée par nombre de traducteurs littéraires n'étant malheureusement pas utilisable dans ce cas!... Nous voulons dire la note en bas de page : "Jeu de mots intraduisible en français ». 
From its birth as an art form Opera has inspired composers and enchanted audiences; it has captivated countries, crossed oceans and conquered continents. It has travelled from our city to your city, a little more than four thousand miles, in a little less than four hundred years.

Today, the time it takes for a great idea to travel between our two cities is quite another tale.

Le titre qui suit emprunte en le modifiant de façon amusante un vers d'une chanson enfantine connue de tous les anglophones et donne un exemple d'asservissement à la typographie. Il est tiré d'une annonce publiée à l'occasion du lancement d'une nouvelle petite voiture, la Véga. Ce nom fera comprendre l'allusion à la chanson en question. Précisons que cette annonce présentait pour la première fois le nom de la voiture. Le titre était composé en gros caractères genre affiche, très gras, de façon à donner une impression de sérieux, de solide. Cette indication typographique a son importance car elle donne le style adopté pour tous les documents de la campagne publicitaire et la nécessité de respecter l'uniformité. Afin qu'on puisse employer un caractère donnant la même impression, le titre français devait avoir sensiblement la même longueur que le titre anglais en plus, naturellement, de se raccrocher au nom de l'étoile Véga. Voici les deux titres :

$\begin{array}{ll}\text { Twinkle } & \text { Une petite } \\ \text { twinkle } & \text { étoile de } \\ \text { little } & \text { première } \\ \text { car. } & \text { grandeur. }\end{array}$

Il y a évidemment perte de l'allusion à la chanson. En revanche, le titre français intrigue, joue sur le mot étoile. (Comme dans l'anglais, il y aura développement de cette idée dans le texte : «Vega. Star of the first magnitude. Brightest in the constellation Lyra. ») Il oppose de plus les mots « petite» et « grandeur ». Toutefois, le manque d'espace a obligé à amputer le titre de quelques mots qui auraient pu en faire un titre dynamique et non statique.

Ce qui précède n'implique pas que le maquettiste doive toujours dicter sa volonté à l'adaptateur, mais il ne faut pas oublier qu'un titre est vu, ou plus exactement perçu, avant d'être lu et que si l'œil est rebuté par une présentation peu plaisante, le lecteur passera outre.

L'exemple qui suit montre un jeu de mots lié à la fois au produit et à l'illustration. Il est tiré d'une annonce pour les timbres-primes «Green Stamps 》. L'illustration représente un service à salade et un timbre-prime. En titre :

$$
\begin{aligned}
& \text { Get } \\
& \text { a salad set } \\
& \text { with greens. }
\end{aligned}
$$

Il est évident que, dans ce cas, toute tentative d'établir une relation entre «timbre vert » ou même «verdure » et l'illustration est vouée à l'échec. Voilà un autre cas où l'adaptateur devra purement et simplement faire ouvre de créateur.

Dernier exemple de difficulté, celle-ci insurmontable : nous avons une locution dans la langue de départ, illustrée, et dont l'équivalence dans la langue 
d'arrivée se fonde sur une autre image. Nous reprenons ici l'excellent exemple cité par ailleurs dans ce numéro (voir p. 40).

En titre d'une annonce pour un cirage : «Clean as a whistle», et comme illustration, un agent de police soufflant dans un sifflet rutilant. La locution française équivalente «propre comme un sou neuf » exigeait évidemment dans ce cas une illustration correspondante, faute de quoi il fallait tout simplement abandonner l'annonce.

\section{Y A-T-IL UN « SECRET DES BONS TITRES »?}

Dans ces deux derniers cas, nous avons atteint les limites de l'adaptabilité. Heureusement, tous les titres ne présentent pas de telles difficultés et la majorité d'entre eux peuvent être rendus sans autres problèmes que ceux qui consistent à leur donner les qualités que nous avons mentionnées... ce qui est déjà assez difficile. En effet, il n'existe pas là de règle d'or qui permette d'atteindre à coup sûr à ces qualités. Voici simplement quelques observations sur le sujet.

Il se peut que l'argument mis en vedette dans le titre semble ne pas convenir au marché de la langue d'arrivée. Il sera peut-être nécessaire d'y substituer un autre argument. Dans ce cas, on procédera avec prudence, après consultation avec les spécialistes du marché, car l'accrochage est une chose délicate.

On se souviendra aussi que le lecteur n'aura pas le titre anglais et le titre français sous les yeux (sauf dans les annonces bilingues, bien entendu, mais elles sont rares - et de toute façon peu recommandables). Il ne faudra donc pas juger le titre français en fonction du titre anglais. Il pourra paraître moins frappant que ce dernier, mais là n'est pas le plus important. Ce qui compte c'est qu'il remplisse bien son office.

Ne pas oublier que le jeu de mots ou l'allusion subtile ou la grosse expression populaire ne sont pas indispensables à l'efficacité du titre. De même, telle trouvaille qui remplit d'aise son auteur n'a pas forcément le même effet sur le lecteur. On se méfiera des titres tellement astucieux qu'ils distraient le lecteur au point que celui-ci est ensuite incapable de les associer à la marque annoncée.

Enfin, ce qui est l'évidence même, mais mérite tout de même d'être dit, il ne faudra pas suivre aveuglément l'anglais, tendance fréquente avec les titres qui se traduisent presque littéralement. S'il est un domaine où il faut se sentir les coudées franches, c'est bien celui du titre. Le but de l'adaptateur sera, là plus que partout ailleurs : faire mieux que l'original. En voici un exemple. Une annonce publiée par une revue féminine américaine propose un tapis à point noué, à faire soi-même ; elle porte en titre :

If you can tie a knot,

you can make these

luxurious, deep-pile

wool rugs.

Une traduction littérale pourrait donner un titre acceptable :

Si vous savez faire

un noud, vous saurez

faire ces somptueux

tapis de haute laine.

Ce titre est construit sur le modèle célèbre «Si vous savez écrire, vous savez dessiner $»$, mais il est lourd, il manque de dynamisme. 
Voyons celui donné à une annonce pour un produit semblable, rédigée en français (ci-dessous la disposition originale, pouvant facilement être modifiée) :

Vous savez faire un noud? Bravo!

Vous savez faire un tapis Pingonin 100\% Léacril.

Ce titre est infiniment plus vivant que le premier, ce qu'il doit en particulier à la forme interrogative et à l'interjection. Il est plaisant (on applaudit une personne parce qu'elle sait faire un nœud). La fin pourrait facilement être modifiée pour rendre luxurious, deep-pile, etc.

Voilà le genre de travail qu'aurait pu faire - et que doit faire - un bon adaptateur en partant du titre anglais.

Encore quelques observations qui peuvent être utiles aux « titreurs ».

Le titre doit atteindre une forte densité de style, c'est-à-dire qu'il doit contenir le maximum de mots pleins pour le minimum de mots outils. C'est une des raisons pour lesquelles le titre est volontiers elliptique, et pas seulement à cause du gain d'espace recherché ${ }^{26}$. La recommandation prend encore plus d'importance avec les titres fleuves que certains annonceurs semblent affectionner. Deux phrases d'une vingtaine de mots chacune, généralement composées de façon dense, doivent en effet être rédigées avec une parfaite clarté, sans mots inutiles, sans idées superflues, si on veut que le lecteur aille jusqu'au bout.

Un titre est rarement une création spontanée. Il doit être travaillé. Voici une façon de procéder qui donne de bons résultats : Lire titre et annonce. Noter tout ce qui se présente à l'esprit, pour le titre et pour le corps de l'annonce. Si l'inspiration ne vient pas, garder le titre pour la fin. Souvent une idée naît pendant la rédaction du corps de l'annonce. Taper les titres qui surgissent spontanément, en respectant la disposition, en coupant selon le sens, en lignes de longueur voulue. Chercher à condenser. Combiner les différentes solutions, s'il y a lieu. Petit à petit, le titre s'élabore. Puis, comme on dit en cuisine, laisser reposer pendant un ou deux jours - si on a le temps - et relire le tout avec des yeux neufs. Dans de tels cas, le travail en équipe est souvent préférable, ou du moins il est bon de présenter son « cuvre » à des personnes qui pourront suggérer d'utiles retouches.

\section{SUITE D'AIDA}

Nous abordons maintenant l'adaptation du corps du texte. Elle présente heureusement beaucoup moins de difficultés que celle du titre.

Le corps de l'annonce a pour rôle de soutenir l'intérêt suscité par le titre (souvenons-nous de la formule AIDA), de provoquer le désir et, dans la plupart des cas, d'exhorter à l'action immédiate.

Soutenir l'intérêt, voilà la condition sine qua non à la lecture de l'annonce. L'adaptateur a là un grand rôle à jouer. L'annonce originale lui apporte un certain nombre d'arguments ordonnés selon une certaine logique, dans une forme, un esprit, un ton propres à convaincre un public donné.

Nous ne reviendrons pas sur la question du choix des arguments. Arrêtonsnous par contre à celle de la logique de leur présentation.

L'ordre de la présentation des arguments, le raisonnement, la démonstration de l'annonce déconcertent souvent l'adaptateur. Il est fréquent que le lecteur francophone considère que l'on saute du coq à l'âne, qu'on fait passer des détails 26. A ce sujet, voir C. R. Haas, Pratique de la publicité, Paris, Dunod, 1969, p. 225ss. 
de second ordre avant des renseignements importants. On note aussi souvent qu'une idée est abandonnée sans être parfaitement développée, puis qu'on y revient. On dira peut-être que ces traits sont le fait de mauvais rédacteurs. Sans doute, mais l'adaptateur ne doit pas, hélas, travailler uniquement sur de bonnes annonces. Il devra donc ordonner toutes ces idées, amener un développement dont l'enchaînement est logique pour le lecteur francophone. Son sens de la langue lui dictera certainement aussi d'une part les divisions en alinéas qui s'imposent et d'autre part les charnières à employer entre les différentes parties de son exposé.

En ce qui concerne la division, l'examen des textes publicitaires anglais amène bien des surprises au lecteur francophone. Les paragraphes sont trop longs ou, à l'inverse - selon une tendance qui se confirme depuis quelques années ils ont trois ou quatre mots seulement. Enfin, les divisions ainsi faites ne correspondent pas aux divisions de l'exposé... ce qu'on retrouve également fréquemment dans les mauvaises adaptations. Que l'adaptateur n'hésite pas, là encore, à remettre de l'ordre dans la maison, en se souvenant que la division en alinéas est nécessaire non seulement du point de vue de la logique de l'exposé, mais aussi du point de vue typographique. Un texte long, d'un seul bloc, a pour effet de rebuter le lecteur. Toutefois, on se gardera de tomber dans l'excès inverse, celui du « micro-alinéa 》 qui exerce le même effet en hachant le texte. "Ce sont les divisions de l'exposé, les étapes de la pensée et du raisonnement qui doivent régir le découpage du texte en paragraphes, et non la simple fantaisie du typographe ${ }^{27} \gg$ (on pourrait ajouter « et du rédacteur de l'annonce originale »).

Pourtant, comme dans le cas du titre, l'adaptateur devra travailler en tenant soigneusement compte de la maquette, afin de ne pas détruire l'harmonie typographique de l'ensemble. Exigences contradictoires, qui ne pourront être satisfaites parfois qu'après de longs tâtonnements ${ }^{28}$.

Les transitions et charnières, presque toujours totalement absentes du texte anglais, doivent aussi retenir toute l'attention de l'adaptateur. Nous ne pouvons mieux faire que de renvoyer le lecteur à l'étude que Vinay et Darbelnet ont fait $\mathrm{du}$ sujet dans leur Stylistique comparée du français et de l'anglais ${ }^{29}$.

Cette question est essentielle et il suffit de lire les adaptations couramment publiées pour se rendre compte que ces textes, calquant l'anglais, sont presque toujours dépourvus des charnières qui les rendraient tellement plus coulants.

De même, encore prisonnier de l'anglais, l'adaptateur craint souvent d'introduire dans son texte des images qui ne se trouvent pas dans l'original; souvent, il n'ose pas non plus employer les procédés de style, répétition, symétrie, antithèse, etc., qui donnent de la vie au texte.

Pourtant, une annonce rédigée simplement, avec un vocabulaire concret et précis, découpée logiquement, s'enchaînant bien, colorée d'images, rythmée, relancée de temps à autre par une figure de style, aura beaucoup plus de chances d'être lue, de jouer le rôle de persuasion qui lui est assigné. Mais cela suppose que l'adaptateur a su s'affranchir totalement des contraintes de l'anglais, souvent contradictoires avec l'esprit de la langue française, et à faire en somme un travail

27. Haas, op. cit., p. 247.

28. Signalons les intéressants exercices de découpage de textes proposés dans $P$. Herbin, Jeux de mots.

29. Vinay et Darbelnet, op. cit., p. 230ss., chap. IV : «Les articulations de l'énoncé ». 
de rédacteur, une fois franchi le cap de la compréhension du message en langue de départ.

De l'annonce, il ne nous reste plus à étudier qu'un des éléments constants : le slogan. L'adaptateur n'aura pas à intervenir pour les autres éléments constants, comme la marque, choisis depuis longtemps lorsque l'annonce lui parviendra. Il pourra simplement mettre en garde l'annonceur contre les assonances ou les connotations défavorables que peut comporter le mot choisi. On rencontre plus souvent que l'on croit des noms de marque de ce genre et nombre de publicitaires québácois peuvent en citer des exemples croustillants... qu'il ne serait pas convenable de rapporter dans ces colonnes.

\section{QUELLE EST VOTRE DEVISE?}

Le slogan... Tout le monde peut se remémorer un certain nombre de ces formules frappantes et brèves, souvent rythmées ou contenant des assonances qui les rendent précisément plus faciles à retenir. Le slogan peut présenter un argument en faveur du produit; il peut définir le produit; il peut tendre à susciter le désir d'acquisition; il peut aussi vanter la marque en ne jouant qu'un rôle de publicité de prestige. D'autres fois, ce sera une formule sans véritable signification, mais qui, répétée à satiété, principalement par les moyens audio-visuels, deviendra une véritable scie, revenant à tout propos dans la conversation des personnes les plus réceptives qui devront naturellement l'associer à la marque ${ }^{30}$.

Le slogan n'est directement trađuisible que dans des cas extrêmement rares et, lorsqu'il l'est, il faut le plus souvent parler bien plus de création que d'adaptation.

Voici un exemple où la traduction du slogan donnerait des résultats pour le moins cocasses (le slogan est tiré de l'annonce pour les timbres-primes citée plus haut) : «S. \& H. Green Stamps - The more you lick them, the more you like them .

Dans l'exemple qui suit, on a pratiqué la traduction avec le résultat qu'on va voir. Le slogan se trouve sur une petite étiquette adhésive fermant une boite de poudre à récurer qui est censée ne pas rayer. Une illustration représente un poussin sortant de l'œuf, «personnage type » de la marque. Le texte est en anglais et en français :

\section{Hasn't scratched yet.}

N'a pas encore égratigné.

Voilà un cas, semblable à ceux rencontrés avec les titres, où l'illustration rend l'adaptation impossible et où le bilinguisme complique encore la situation.

Un examen rapide des numéros de novembre 1971 du Reader's Digest et de sa version française Sélection nous a pcrmis de relever un certain nombre de slogans anglais et de leur version française. On verra les divers procédés

30. Il est amusant de rapprocher l'usage ainsi fait de ces formules avec certaines manifestations de l'aliénation du langage décrites par le $D^{r} P$. Chauchard : "Ce sont [...] les séries de mots assonancés et les calembours à caractère forcé dans un contexte aberrant, la répétition de mots en écho ou le recours incessant à certaines phrases ou locutions, l'émission incessante de litanies dépourvues de sens $»$ (le Langage et la pensée, Paris, Presses Universitaires de France, 1970, p. 99, chap. II, "Idiots et aliénés ; l'aliénation du langage »). Ne croirait-on pas lire la description de certaines annonces télévisées ? 
employés par les adaptateurs. Pour un désodorisant (ou un déodorant, si on préfère) :

Ban Roll-on for confidence.

Ban à bille... question de confiance.

Le mot clé "confiance» reste le même, mais il y a certainement gain en français par l'emploi d'une formule lexicalisée " question de confiance ».

Pour une marque d'appareils électroménagers :

Hoover... We work hard to make your housework easy.

Hoover... Nous travaillons dur pour vous faire la vie plus douce.

Traduction presque littérale du slogan anglais, conservant l'antithèse hard-easy avec le couple «dur-douce». Le slogan français ne compte qu'un mot de plus que le slogan anglais.

Maintenant, le slogan, employé depuis longtemps déjà, d'une marque de teinture pour les cheveux :

Does she or doesn't she?

Oui ou non... Qui sait?

(Slogan complété par : «Seul son coiffeur le sait».) Voilà un cas d'adaptation bien réussie, avec cinq mots courts, faciles à mémoriser et qui rendent bien l'idée voulue par le texte original, à savoir que, avec ce produit, on ne peut déceler si les cheveux ont été teints ou non.

Dernier exemple, pour lequel nous ne possédons pas le texte anglais, mais qu'il est intéressant de comparer avec un slogan connu, exprimant exactement la même idée. Une marque de pneus annonce la garantie suivante : "Vous roulez dans la neige ou General paie votre remorquage. » Phrase banale qui n'a guère de chance d'être retenue. À l'opposé, le slogan des pneus Firestone, plus elliptique, mieux rythmé, dont les deux termes riment, a beaucoup plus de chance de l'être :

You go, or we pay the tow.

Vous roulez... ou le remorquage est payé.

Ces quelques exemples sont loin d'épuiser le sujet, car les procédés employés dans la «fabrication» des slogans varient presque à l'infini. Au lecteur désirant apprendre plus sur le sujet, nous conseillons de consulter les deux ouvrages déjà cités : Pratique de la publicité, de C. R. Haas et Manuel pratique de publicité, de P. Herbin.

\section{LE PUBLICITAIRE : UN HOMME TOUJOURS EN ÉVEIL}

Nous touchons à la fin de ce survol de l'adaptation publicitaire. Il reste certainement encore beaucoup à dire. Chacun des sujets effleurés ici demanderait un développement détaillé. En outre, nous n'avons examiné qu'un des moyens de publicité : l'annonce, parce que c'est le plus typique. Il en reste bien d'autres, on le sait, qui présentent des difficultés d'adaptation propres. Toutefois, les techniques générales d'adaptation restent les mêmes que celles de l'annonce : le dépliant peut être traité comme une annonce; la page de catalogue aussi, qui amplifie toutefois les problèmes de l'espace; le texte du panneau-réclame est généralement un slogan. Mentionnons aussi les annonces radiodiffusées et télévisées, pour mémoire seulement, car elles relèvent de techniques bien différentes de celles de la publicité imprimée. 
Nous terminerons sur une simple observation. En fait, le travail de l'adaptateur n'a jamais de fin. Le publicitaire doit constamment se tenir au courant de la réclame qui se fait dans les journaux, à la radio, à la télévision. Il doit y déceler les tendances nouvelles, y puiser des sujets d'inspiration (aucune création n'est absolument vierge d'influences extérieures). Il doit aussi être au courant de l'actualité, de l'évolution des goûts du public; il doit être à l'affût des expressions nouvelles, de tout ce qui pourra, à l'occasion, capter l'attention du lecteur ou de l'auditeur. La publicité étant omniprésente, c'est dire que son esprit ne sera jamais en repos.

Mais la recommandation la plus importante qu'on puisse lui faire est peut-être celle-ci : savoir se mettre à la place du public auquel il s'adresse, savoir considérer le texte qu'il écrit avec les yeux de celui qui le lira. L'empathie est une des qualités essentielles du publicitaire.

Ainsi pourra-t-il réussir cette entreprise qui consiste en somme à prendre une personne dont on connaît à peu près la taille, les goûts en matière de coupe et de couleur et les habitudes vestimentaires, et à lui faire accepter un habit fait sur mesure pour quelqu'un d'autre.

ROGER BOIVINEAU 